\title{
From first to second wave: follow-up of the prospective COVID-19 cohort (KoCo19) in Munich (Germany)
}

Katja Radon ${ }^{1,2,3^{*}+} \mathbb{0}$, Abhishek Bakuli ${ }^{4 \dagger}$, Peter Pütz ${ }^{5,6 \dagger}$, Ronan Le Gleut ${ }^{5,7 \dagger}$, Jessica Michelle Guggenbuehl Noller ${ }^{4}$, Laura Olbrich ${ }^{4,8}$, Elmar Saathoff ${ }^{4,8}$, Mercè Garí ${ }^{5}$, Yannik Schälte ${ }^{5,9}$, Turid Frahnow ${ }^{5,6}$, Roman Wölfel ${ }^{8,10}$, Michael Pritsch ${ }^{4,8 \dagger}$, Camilla Rothe ${ }^{4}$, Michel Pletschette ${ }^{4}$, Raquel Rubio-Acero ${ }^{4}$, Jessica Beyerl ${ }^{4}$, Dafni Metaxa ${ }^{4}$, Felix Forster ${ }^{1,3}$, Verena Thiel ${ }^{4}$, Noemi Castelletti ${ }^{4}$, Friedrich Rieß ${ }^{4,8}$, Maximilian N. Diefenbach ${ }^{4}$, Günter Frösch ${ }^{2,4}$, Jan Bruger ${ }^{4}$, Simon Winter ${ }^{4}$, Jonathan Frese ${ }^{4}$, Kerstin Puchinger ${ }^{4}$, Isabel Brand ${ }^{4}$, Inge Kroidl ${ }^{4,8}$, Andreas Wieser ${ }^{4,8+}$, Michael Hoelscher ${ }^{2,4,8+}$, Jan Hasenauer ${ }^{5,9,11 \dagger}$ and Christiane Fuchs ${ }^{5,6,7,9+}$ on behalf of the KoCo19 study group

\begin{abstract}
Background: In the 2nd year of the COVID-19 pandemic, knowledge about the dynamics of the infection in the general population is still limited. Such information is essential for health planners, as many of those infected show no or only mild symptoms and thus, escape the surveillance system. We therefore aimed to describe the course of the pandemic in the Munich general population living in private households from April 2020 to January 2021.

Methods: The KoCo19 baseline study took place from April to June 2020 including 5313 participants (age 14 years and above). From November 2020 to January 2021, we could again measure SARS-CoV-2 antibody status in 4433 of the baseline participants (response 83\%). Participants were offered a self-sampling kit to take a capillary blood sample (dry blood spot; DBS). Blood was analysed using the Elecsys ${ }^{\circledR}$ Anti-SARS-CoV-2 assay (Roche). Questionnaire information on socio-demographics and potential risk factors assessed at baseline was available for all participants. In addition, follow-up information on health-risk taking behaviour and number of personal contacts outside the household $(N=2768)$ as well as leisure time activities $(N=1263)$ were collected in summer 2020.

Results: Weighted and adjusted (for specificity and sensitivity) SARS-CoV-2 sero-prevalence at follow-up was 3.6\% (95\% Cl 2.9-4.3\%) as compared to $1.8 \%(95 \% \mathrm{Cl} 1.3-3.4 \%)$ at baseline. $91 \%$ of those tested positive at baseline were also antibody-positive at follow-up. While sero-prevalence increased from early November 2020 to January 2021, no indication of geospatial clustering across the city of Munich was found, although cases clustered within households. Taking baseline result and time to follow-up into account, men and participants in the age group 20-34 years were
\end{abstract}

\footnotetext{
*Correspondence: katja.radon@med.Imu.de

${ }^{\dagger}$ Katja Radon, Abhishek Bakuli, Peter Pütz, Ronan Le Gleut contributed

equally

${ }^{\dagger}$ Andreas Wieser, Michael Hoelscher, Jan Hasenauer, Christiane Fuchs

contributed equally

${ }^{1}$ Institute and Outpatient Clinic for Occupational, Social

and Environmental Medicine, University Hospital, LMU Munich,

80336 Munich, Germany

Full list of author information is available at the end of the article
}

(C) The Author(s) 2021. Open Access This article is licensed under a Creative Commons Attribution 4.0 International License, which permits use, sharing, adaptation, distribution and reproduction in any medium or format, as long as you give appropriate credit to the original author(s) and the source, provide a link to the Creative Commons licence, and indicate if changes were made. The images or other third party material in this article are included in the article's Creative Commons licence, unless indicated otherwise in a credit line to the material. If material is not included in the article's Creative Commons licence and your intended use is not permitted by statutory regulation or exceeds the permitted use, you will need to obtain permission directly from the copyright holder. To view a copy of this licence, visit http://creativecommons.org/licenses/by/4.0/. The Creative Commons Public Domain Dedication waiver (http://creativeco mmons.org/publicdomain/zero/1.0/) applies to the data made available in this article, unless otherwise stated in a credit line to the data. 
at the highest risk of sero-positivity. In the sensitivity analyses, differences in health-risk taking behaviour, number of personal contacts and leisure time activities partly explained these differences.

Conclusion: The number of citizens in Munich with SARS-CoV-2 antibodies was still below $5 \%$ during the 2 nd wave of the pandemic. Antibodies remained present in the majority of SARS-CoV-2 sero-positive baseline participants. Besides age and sex, potentially confounded by differences in behaviour, no major risk factors could be identified. Non-pharmaceutical public health measures are thus still important.

Keywords: COVID-19, SARS-CoV-2, Population-based cohort study, Sero-prevalence, Sero-incidence, ORCHESTRA

\section{Background}

The SARS-CoV-2 virus affected almost all nations within a few weeks. Given the nature of the virus, a large proportion of infected individuals present only mild symptoms or no symptoms at all. Therefore, population-based sero-prevalence studies are necessary to estimate the true prevalence of the infection in the population. Starting in March 2020, such sero-prevalence studies have been conducted in many countries, mostly during or after the first wave of the pandemic [1]. Depending on the serological test used, the type of sample drawn, the timing of the study, and the region, general population sero-prevalence ranged from $<0.1 \%$ in Brazil to well over $20 \%$ in the USA [2]. For the German context, we reported a sero-prevalence of $1.8 \%$ in Munich, sampled towards the end of the first wave in Germany [3].

Following the introduction of public health measures (lock-down including school closures) in March 2020 in Germany, the first wave of the pandemic was perceived as relatively mild with around 6000 cases registered in Munich during this period (Munich population $1.5 \mathrm{Mio}$ ). Between June and October, public health measures were reduced, although physical distancing of $1.5 \mathrm{~m}$ between two persons, avoidance of mass events, and obligatory use of face masks, e.g. in restaurants and shops, were still required. Subsequently, officially registered monthly case numbers in Munich rose from 389 in June to 7181 in October 2020. A partial national lockdown was implemented on November 2nd, 2020. After a further rise in officially registered case numbers and COVID-19 related deaths, national lock-down measures were increased from December 16th, 2020 on, including closure of schools, shops (other than grocery and drug stores), restaurants, and hotels.

Given that asymptomatic and mildly symptomatic cases escape surveillance systems, prospective population-based cohort studies offer the chance to better understand the course of disease in the general population. They are independent of testing strategies and help to identify the population at risk over time. In addition, they provide an indication of population groups less well protected by public health measures. We therefore followed up the participants of the Munich COVID-19 cohort (KoCo19) to explore the SARS-CoV-2 antibody prevalence in the Munich general population at two time points: at the time the acute outbreaks happened and seven months later. In addition, we aimed at the identification of risk factors (demographic, social-economic, health status or individual risk behaviours factors) for acquiring SARS-CoV-2 infection defined by serology. The baseline study took place from April to June 2020, the questionnaire follow-up in summer 2020 and the 1st antibody follow-up was realised from early November 2020 to January 2021. On December 1st 2020 the KoCo19 cohort joined the ORCHESTRA (Connecting European Cohorts to Increase Common and Effective Response to SARS-CoV-2 Pandemic) project.

\section{Methods \\ Study population and field work Baseline SARS-CoV-2 antibody and questionnaire study}

We described the baseline study in detail in [4]. In short, a random sample of the Munich population living in private households was drawn by random walk method. All household members older than 13 years were invited to provide a serum sample and to answer an online questionnaire. Serum samples were analysed for SARS-CoV-2 antibodies using the Elecsys ${ }^{\circledR}$ Anti-SARS-CoV-2 (Roche) test [5]. Field work for the baseline study took place between April 5th and June 12th, 2020.

\section{Questionnaire follow-up}

An online questionnaire covering risk behaviour, health related items, and psychosocial aspects (hereafter "behaviour questionnaire") was offered from June 4th to October 31st, 2020 to all 5240 participants who did not withdraw from the study. In parallel, an online-questionnaire on leisure time behaviour was available (hereafter "leisure time questionnaire"). We split the questionnaire into two, because long questionnaires are less likely to be completed [6]. Participants recruited in April (May to June) 2020 received an invitation via e-mail on June 4th (June 25th) with subsequent reminders and telephone follow-ups. In total, 3400 participants completed the behaviour questionnaire and 1390 participants the leisure time questionnaire. 


\section{1st SARS-CoV-2 antibody follow-up}

On November 2nd 2020, we started the 1st antibody follow-up by sending out boxes with a self-sampling kit to take a capillary blood samples (dry blood spot; DBS) to the 5292 participants (2978 households) of the baseline study. Between baseline and follow-up, 77 participants withdrew from the study and were thus not contacted for the follow-up. Instructions for selfsampling were provided, including a video tutorial (https://www.youtube.com/watch?v=vpZUzuQV10 $E \&$ feature $=$ emb_title). Samples were collected using a barcode-labelled neonatal screening filter card (Euroimmun ZV 9701-0101) with circles indicating where the blood should be collected. Afterwards, participants should dry the filter card at least $12 \mathrm{~h}$ at room temperature, pack them in the sealable plastic pouch, place the plastic pouch into the prepaid envelope, and ship the envelope by mail to the laboratory. In case of handling difficulties, our telephone and e-mail hotline were available for any questions.

From November 2nd to January 31st, 2021, we received 4444 DBS samples from 2571 households (individual response $84 \%$, household response $86 \%$ ). Roughly half of the DBS samples (2372 of 4433; 54\%) arrived at our laboratory within 1 week of mailing (November 2nd to November 8th). By week 2 (November 9th to November 15th), more than three quarters were received (3369 of 4433; 76\%). Most of the remaining samples were turned in between week $3(\mathrm{~N}=372$ from November 16th to November 22th) and week $4(\mathrm{~N}=343$; November 23rd to November 29th). Few samples were received in December 2020 and January 2021 ( $\mathrm{N}=326$; 7\%). Participants not being able to collect a DBS on their own $(\mathrm{N}=29)$ and those with intermediate results $(\mathrm{N}=34$, s. laboratory methods) were offered a full-blood test at our centre. For the latter group, this served to clarify the DBS result. However, 11 of the 34 participants with intermediate results in the DBS did not show up at our centre and thus had to be excluded from analyses, leaving 4433 subjects with baseline questionnaire, baseline serology and follow-up DBS data for the main analyses (Fig. 1).

\section{Questionnaire data}

The following items were considered for the analyses presented in this paper:

Baseline individual questionnaire:

- Socio-demographics: age, sex (male, female), schooling ( $<12$ years, $\geq 12$ years, in school), current job (employed, self-employed, not working (unemployed, retired, parental leave, sabbatical, students), others (voluntary social year, military service, part-time job-

ber, reduced working hours))

- Country of birth: Germany, others

- Smoking: current, ex, never smokers

- Chronic conditions: diabetes, cardiovascular diseases, autoimmune diseases, respiratory diseases (yes vs no)

- General health: "In general, how would you rate your health" assessed on a five point Likert scale from poor to excellent. As very few participants reported "poor", the poor and fair category were combined.

Baseline household questionnaire:

- Household size: 1, 2, 3-4,>5 inhabitants

- Household income: $\leq 2500 €, 2500-\leq 4000 €$, $4000-\leq 6000 €,>6000 €$

- Living area per inhabitant: $\leq 30 \mathrm{sqm}, 30-\leq 40 \mathrm{sqm}$, $40-\leq 55 \mathrm{sqm},>55 \mathrm{sqm}$

- Household type: single, couple, family, others (shared apartments by e.g., students, subleasing, and assisted accommodation)

- Housing type: building with 1-2 apartments, 3-4 apartments, $\geq 5$ apartments

Follow-up questionnaire:

- Self-estimated health-related risk taking behaviour (10-level Likert scale from "not at all risk tolerant" to "very risk tolerant"): Dichotomised into not high $(\leq 5$, Quartile 3$)$ and high self-estimated healthrelated risk taking behaviour $(>5)$

- Personal contacts: Five questions on places of personal contacts outside the own household during the two weeks before answering the questionnaire (meeting people, grocery shopping, shopping, use of public transport, work outside home), each assessed on a 5-level Likert scale: not at all $(=1)$; once per week $(=2)$; $2-4$ times per week $(=3)$; 5 times per week $(=4)$; more often $(=5)$. Places of personal contacts were multiplied by frequency of contacts $(0$ contacts $(=0), 1$ contact $(=1), 2-4$ contacts $(=2)$ and $5+$ contacts $(=3)$ ) and summed up, resulting in a score ranging from 0 to 25 . The score was dichotomised into lower number of personal contacts $(\leq 8$, Median) and higher number of personal contacts $(>8)$. The score was dichotomised into non-high leisure time activities $(\leq 11$, Quartile 3$)$ and high leisure time activities $(>11)$. 


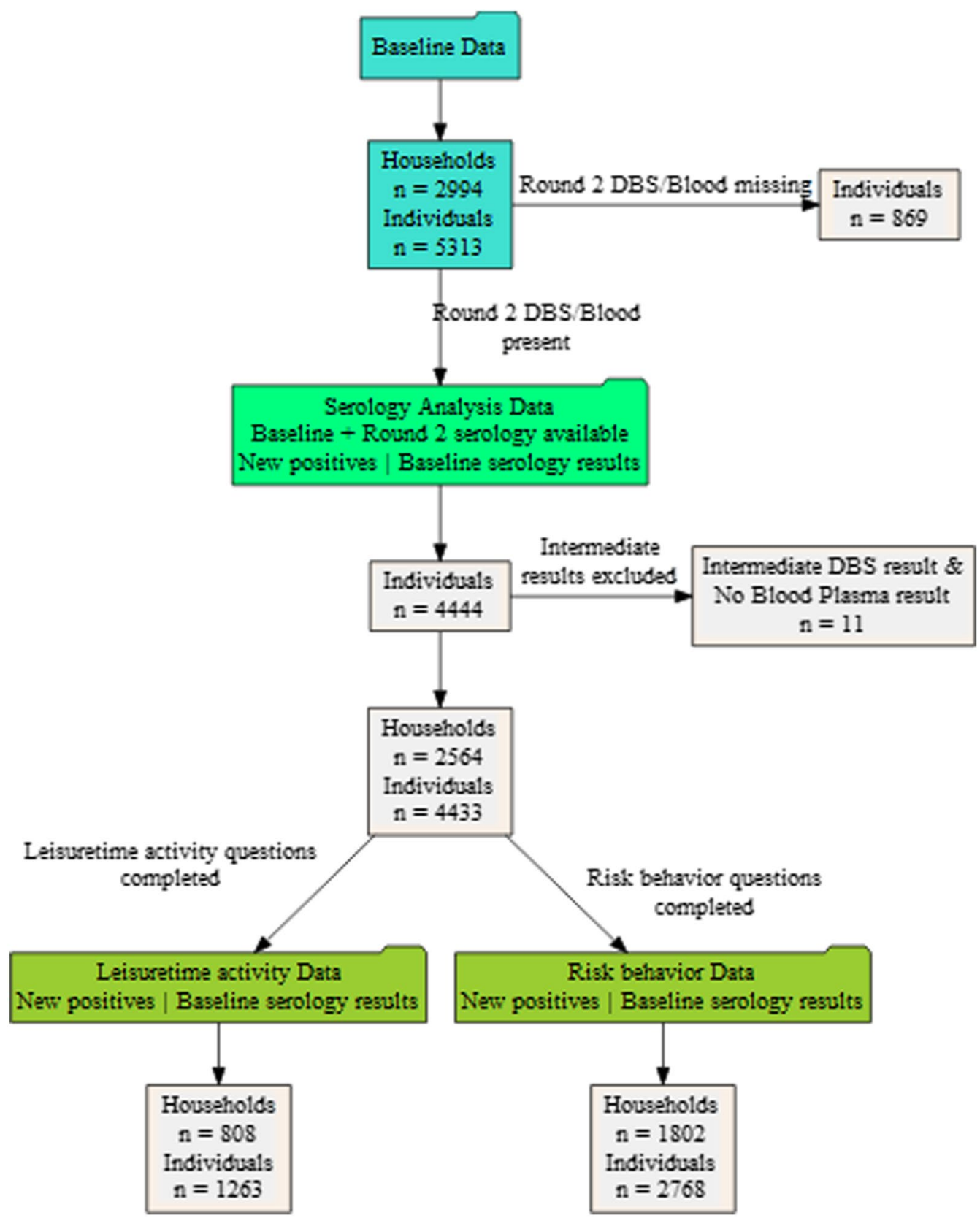

Fig. 1 Flow chart of obtaining the study population. Light blue boxes indicate the total number of participants and households in the baseline study, the light green box refers to subjects included in the sero-prevalence analyses of the data, dark green boxes indicate analyses restricted to sero-incidence data for subjects with complete leisure time information (left) and risk behaviour data (right)

- Number and intensity of leisure time activities before the pandemic (in February 2020): For that time, 16 activities assessed on a 5-level Likert scale from "never" $(=0)$ to "very often" $(=4)$ : visit family member; visit friends; going out with friends; attend a party, festival, bar, pub or disco; go to the cinema; attend a theatre, opera or ballet performance; work out in a gym; visit a swimming pool; visit a sauna; skiing; train for a team sport or take part in sporting competitions; watch a sports game or event live outdoors; watch a sports game or event live indoors; worship attendance; play an instrument in an orchestra; sing in a choir. Activities were multi- 
plied by the Likert scores and summed up resulting in a score from 0 to 64 .

- Number and intensity of leisure time activities two weeks prior to the follow-up questionnaire: The score for leisure time activities at follow-up was built the same way as the score for leisure time activities before the pandemic. However, the number of leisure time activities was only seven at that time as many activities were not possible due to the restrictions related to the pandemic: visit family member; visit friends; going out with friends; visit a swimming pool; worship attendance; play an instrument in an orchestra; sing in a choir. Therefore, the resulting score only ranged from 0 to 28 . The score was dichotomised into non-high leisure time activities at follow-up ( $\leq 5$, Quartile 3$)$ and high leisure time activities $(>5)$.

\section{Laboratory method and cross-validation with blood samples}

Filter paper cards were further processed if at least two of the five circles on the card were completely soaked with blood. Valid samples were stored at $4{ }^{\circ} \mathrm{C}$ until analysis. Before analysis, filter paper cards were equilibrated to room temperature and three blood-soaked smaller circles (diameter $3.2 \mathrm{~mm}$ ) of each filter paper card were automatically punched into a 96-wells plate (PantheraPuncher $^{\text {TM }}$ 9, PerkinElmer). After elution, samples were transferred to a Cobas e801 module (Roche) compatible sample micro cup (Roche, 05085713001) for analysis using the Elecsys ${ }^{\circledR}$ Anti-SARS-CoV-2 assay (Roche). Based on our validation study, DBS samples were considered positive if SARS-CoV-2 antibody levels were $\geq 0.12$. Samples with SARS-CoV-2 antibody levels in the range between 0.09 and 0.12 were considered intermediate, and subsequently confirmed by plasma samples (s. Study population and field work). All other samples were considered negative. Compared to full blood samples, sensitivity of the DBS method was $99.2 \%$ and specificity $98.7 \%$. Details of the laboratory methods are described in [7].

\section{Statistical analyses}

All statistical analyses were performed using the statistical software R (version 4.0.3, R Development Core Team, 2020).

The SARS-CoV-2 sero-prevalence was estimated primarily based on the DBS test results of the study participants applying the classification as described above (Laboratory methods). If the DBS test yielded an intermediate result, we considered the result of the full blood sampling. As described in [5], an optimised cut-off of 0.4218 for the full blood sampling was used to predict
SARS-CoV-2 sero-positivity with an estimated specificity and sensitivity of $99.7 \%$ and $88.6 \%$, respectively (with regard to PCR test results considered as ground truth). We used these estimates to adjust the prevalence for the imperfect test performance [8]. The specificity and sensitivity of DBS with regard to full blood samples being very high, additional adjustment was omitted (Additional file 1: Appendix Text and Table S1).

The prevalence (adjusted or unadjusted for the specificity and the sensitivity of the test) was calculated in two different ways: including the information from the sampling design of the cohort [3] via the use of a weighting scheme, or without it. To account for the sampling design, the sampling weights computed at baseline (inverse of the probability of each individual to be included in the sample) were used for the follow-up analysis. These sampling weights were corrected for the attrition observed at follow-up by modelling the underlying non-response mechanism and estimating probabilities of response for each unit. Ten response homogeneity groups (where we assumed the non-response to be completely at random, [9]) were created using the deciles of the estimated probabilities of response. These weights adjusted for the non-response were calibrated [10] on updated information from the Munich population (at 31.12.2020) in order to mirror the age, sex, country of birth, presence of children in the household and single member household structures. Moreover, to correct the sample for the loss of positive cases at follow-up, the sampling weights were calibrated on the estimated number of positive cases at baseline. Weighted prevalence estimates were calculated using these calibrated weights, and the associated 95\% confidence intervals were computed based on variance estimators based on linearization [10] and residual $[10,11]$ techniques. These variance estimates were computed in order to account for every step in the selection process of the units, i.e., $\mathrm{V}=\mathrm{V} 1+\mathrm{V} 2$ with $\mathrm{V} 1$ the variance due to the sampling design and V2 the one due to the non-response [12]. For unweighted prevalence estimates, confidence intervals were determined by using a nonparametric cluster bootstrap procedure that accounts for household clustering [13]. To that end, 5000 bootstrap datasets were generated each by sampling $n_{h}$ households with replacement from the original sample of $n_{h}$ households. The sero-prevalence was estimated in each bootstrap sample and the 2.5 and 97.5 percentiles of the resulting 5000 estimates defined the $95 \%$ confidence intervals.

To analyse spatial clustering, we considered the mean within-cluster variance of the binary test results, with cluster variables being households, buildings, and geospatial clusters of different sizes. We performed a nonparametric approximate permutation test with 10,000 
random permutations of cluster assignments. To account for household clustering, only full households were permuted when considering buildings and geospatial clusters [14]. In addition to this, we performed borough level sero-prevalence mapping using Conditional Auto Regressive Models which account for the spatial autocorrelation among neighbouring boroughs by using random effects. This allowed us to investigate if sero-prevalence was associated with the population density or not, as well as obtaining Borough/District level estimates within the city of Munich (Additional file 1: Appendix 2) [15-18].

We used generalised linear mixed models (GLMMs using the logit link function) to analyse the association between potential risk factors and SARS-CoV-2 sero-positivity at 1 st follow-up, with a random effect for households to account for within household clustering of the data. Odds Ratio estimates and the corresponding confidence intervals were obtained applying a Bayesian framework with uniform priors on the regression estimates using the brms (Bayesian Regression Models using 'Stan') package in $R[19,20]$. To account for missing data in covariates, we used the Joint Analysis and Imputation of Incomplete Data Framework (JointAI) in R for sensitivity analyses [21, 22]. In these sensitivity analyses, broad normal priors with mean zero and standard deviation 100 were used. The regression estimates were adjusted for the SARS-CoV-2 serology results at baseline, the time elapsed since baseline visit, age, and sex of the individual. Essentially, this adjustment for baseline positivity allowed us to obtain risk factors associated with newly incident cases within our cohort over and above the baseline positives.

To explore the importance of behavioural factors and leisure-time activities for the incidence of infection between baseline and follow-up, we used data of the 1st questionnaire follow-up combined with the DBS results. For these analyses, we included information of 2768 participants who responded to the behaviour questionnaire and had serology results; for the leisure time activities, we had questionnaire information for 1263 persons with serological results. Due to the large proportion of missing questionnaire data, we restricted these analyses to complete data and aggregated at the levels of the outcome variables. We analysed the incidence of new SARS$\mathrm{CoV}-2$ infections (as binomial outcome for proportions) between baseline and follow-up stratified for risk behaviour, leisure time activities, sex and age, using the count of new positives among the observed. Similar models were also applied to evaluate the association of the population densities at the constituency level and the trend in sero-prevalence estimates using aggregated data.

\section{Results}

Non-responder analysis

Follow-up participants compared to participants lost to follow-up were more likely to be between 35 and 79 years old, born in Germany ( $84 \%$ vs. $74 \%$ ), and to have a higher socio-economic status (Table 1). The latter was indicated, e.g., by level of education, household income, living area, and type of building. In addition, the sero-prevalence of SARS-CoV-2 at baseline was lower among follow-up participants (1.6\%) compared to baseline only participants (2.6\%; Table 1). These losses of positive cases at follow-up led to an underestimation of the total number of people tested positive at baseline in Munich (22,064 vs. 25,900 using all participants at baseline). To correct for this attrition bias, the weights at follow-up were calibrated on the estimate of positive cases at baseline, in addition to the other margins used to mirror the Munich structure.

\section{SARS-CoV-2 sero-prevalence over time}

The overall weighted and adjusted (for specificity and sensitivity) SARS-CoV-2 sero-prevalence at followup was 3.6\% (95\% CI 2.9-4.3\%; Fig. 2). The overall unweighted and adjusted sero-prevalence was 3.1\% (95\% CI 2.5-3.8\%), increasing from $2.5 \%$ (95\% CI 1.7-3.3\%) in the first week of November to $4.0 \%$ (95\% CI 1.6-6.8\%) in the last week of November (Fig. 3). About half of the participants with intermediate result in the DBS test had a positive test result when considering the plasma sample. As plasma samples were collected in December and January, the prevalence estimates in the latest weeks were artificially high. Yet, the overall upward trend remained after excluding the participants with intermediate DBS result (Additional file 1: Figures S1, S2).

Most participants who were SARS-CoV-2 sero-positive at baseline continued to be sero-positive at follow-up (64 out of 70 sero-positive subjects at baseline; Additional file 1: Table S3). The weighted and adjusted SARS-CoV-2 sero-incidence (negative at baseline, positive at followup) was estimated at 1.6\% (95\% CI 0.95-2.3\%) (Fig. 2).

\section{Geospatial distribution of SARS-CoV-2 sero-prevalence}

Looking at the geospatial distribution of SARS-CoV-2 sero-prevalence by Munich city boroughs (Fig. 4), an increase was visible from the South-East to the NorthWest of the city, although these differences were rather small. The estimates of Moran's I for spatial autocorrelation was 0.015 using the continuous distance based spatial neighbourhood matrix resulting in a p-value of 0.304 , and hence was not statistically significant. Using a binary spatial neighbourhood matrix, the estimated Moran's I was 0.025 with a corresponding p-value of 0.269 - thereby the conclusion remained unchanged. We 
Table. 1 Descriptive data of the KoCo-19 follow-up participants in comparison to participants only taking part in the baseline study ("Losses to follow-up")

\begin{tabular}{|c|c|c|c|c|c|c|c|c|}
\hline \multirow[t]{2}{*}{ Variable } & \multirow[t]{2}{*}{ Categories* } & \multirow[t]{2}{*}{$\mathbf{N}$} & \multirow[t]{2}{*}{$\mathrm{n}_{\text {Missing }}$} & \multicolumn{2}{|c|}{$\begin{array}{l}\text { Losses to follow-up } \\
(\mathrm{N}=\mathbf{8 8 0})\end{array}$} & \multicolumn{2}{|c|}{$\begin{array}{l}\text { Follow-up participants } \\
(\mathrm{N}=4433)\end{array}$} & \multirow[t]{2}{*}{$\mathbf{p}^{\#}$} \\
\hline & & & & $\mathbf{n}$ & $\%$ & $\mathbf{n}$ & $\%$ & \\
\hline & Total & 5313 & 0 & 880 & 16.6 & 4433 & 83.4 & \\
\hline Sex & Female & 2766 & 0 & 446 & 50.7 & 2320 & 52.3 & 0.38 \\
\hline Age & $0-19$ & 267 & 0 & 55 & 6.3 & 211 & 4.8 & $<0.001$ \\
\hline \multirow[t]{5}{*}{ (years) } & $20-34$ & 1346 & & 306 & 34.8 & 1040 & 23.5 & \\
\hline & $35-49$ & 1542 & & 271 & 30.8 & 1271 & 28.7 & \\
\hline & $50-64$ & 1306 & & 140 & 15.9 & 1166 & 26.3 & \\
\hline & $65-79$ & 676 & & 77 & 8.8 & 599 & 13.5 & \\
\hline & $80+$ & 176 & & 31 & 3.5 & 145 & 3.3 & \\
\hline Birth country & Germany & 3999 & 465 & 478 & 73.9 & 3521 & 83.8 & $<0.001$ \\
\hline \multirow[t]{3}{*}{ Level of education } & Student & 100 & 701 & 20 & 3.2 & 80 & 2.0 & 0.01 \\
\hline & $<12$ years & 1386 & & 211 & 33.8 & 1175 & 29.5 & \\
\hline & $\geq 12$ years & 3126 & & 394 & 63.0 & 2732 & 68.5 & \\
\hline Occupationally active & Yes & 3935 & 470 & 522 & 80.8 & 3413 & 81.3 & 0.75 \\
\hline \multirow[t]{3}{*}{ Smoking status } & Never smoker & 2540 & 487 & 323 & 50.0 & 2217 & 53.0 & 0.007 \\
\hline & Ex-smoker & 1411 & & 177 & 27.4 & 1234 & 29.5 & \\
\hline & Current smoker & 875 & & 146 & 22.6 & 729 & 17.5 & \\
\hline \multirow[t]{4}{*}{ General health } & Excellent & 798 & 466 & 112 & 17.3 & 686 & 16.4 & 0.20 \\
\hline & Very good & 2126 & & 274 & 42.3 & 1852 & 44.1 & \\
\hline & Good & 1717 & & 224 & 34.6 & 1493 & 35.5 & \\
\hline & Not good & 206 & & 37 & 5.7 & 169 & 4.0 & \\
\hline Respiratory allergies & Yes & 1379 & 540 & 187 & 29.2 & 1192 & 28.8 & 0.85 \\
\hline Diabetes & Yes & 208 & 504 & 41 & 6.4 & 167 & 4.0 & 0.009 \\
\hline CVD & Yes & 892 & 513 & 91 & 14.2 & 801 & 19.3 & 0.002 \\
\hline Obesity & Yes & 279 & 521 & 31 & 4.9 & 248 & 6.0 & 0.28 \\
\hline \multirow[t]{4}{*}{ Household type } & Single & 680 & 494 & 92 & 14.4 & 588 & 14.1 & $<0.001$ \\
\hline & Couple & 1705 & & 174 & 27.2 & 1531 & 36.6 & \\
\hline & Family & 1953 & & 279 & 43.6 & 1674 & 40.1 & \\
\hline & Others & 481 & & 95 & 14.8 & 386 & 9.2 & \\
\hline Household income & $\leq 2500$ & 593 & 1636 & 92 & 20.3 & 501 & 15.5 & 0.02 \\
\hline \multirow[t]{3}{*}{ (Euro) } & $2501-4000$ & 817 & & 111 & 24.4 & 706 & 21.9 & \\
\hline & $4001-6000$ & 1176 & & 133 & 29.3 & 1043 & 32.4 & \\
\hline & $6000+$ & 1091 & & 118 & 26.0 & 973 & 30.2 & \\
\hline Living area/inhabitant & $\leq 30$ & 1702 & 513 & 270 & 42.5 & 1432 & 34.4 & $<0.001$ \\
\hline \multirow[t]{3}{*}{ (sqm/individual) } & $31-40$ & 1213 & & 175 & 27.5 & 1038 & 24.9 & \\
\hline & $41-55$ & 988 & & 99 & 15.6 & 889 & 21.3 & \\
\hline & $55+$ & 897 & & 92 & 14.5 & 805 & 19.3 & \\
\hline Building type & $1-2$ & 1433 & 0 & 170 & 19.3 & 1263 & 28.5 & $<0.001$ \\
\hline \multirow[t]{3}{*}{ (No of apartments) } & $3-4$ & 354 & & 47 & 5.3 & 307 & 6.9 & \\
\hline & $5+$ & 3519 & & 663 & 75.3 & 2856 & 64.4 & \\
\hline & Others & 7 & & 0 & 0.0 & 7 & 0.2 & \\
\hline Baselinesero-prevalence & Positive & 93 & & 23 & 2.6 & 70 & 1.6 & 0.047 \\
\hline
\end{tabular}

*For category definitions please see the methods section of the article

\# p-values were obtained by Chi Square Test using 10,000 replicates for variables with more than 2 categories. For variables with only 2 categories, p-values were obtained by Fisher Exact Test 


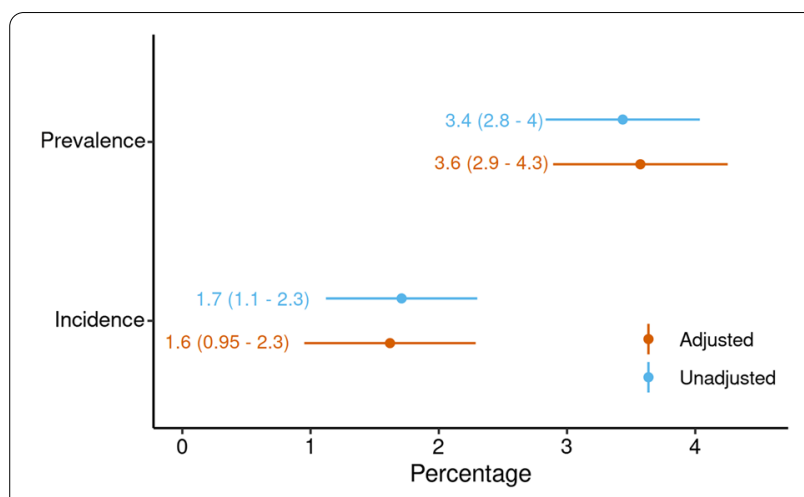

Fig. 2 Weighted sero-prevalence and sero-incidence in \% at follow-up adjusted (orange) and unadjusted (blue) for test specificity and sensitivity. The unadjusted weighted sero-prevalence was 3.4\%, the relative number of new cases between baseline and follow-up $1.7 \%$. Adjustment only slightly changed the unadjusted results

also took population density as a potential risk factor into account and could not find any statistically significant association between population density in the constituency and SARS-CoV-2 sero-prevalence (Additional file 1: Figure S3).

\section{Risk factors for SARS-CoV-2 sero-prevalence}

The distribution of SARS-CoV-2 sero-positivity by covariates is shown in Additional file 1: Table S3. Taking household clustering, time elapsed between baseline and follow-up, and baseline result into account, men had statistically significantly higher odds of sero-positivity at follow-up (OR adjusted for age: 2.4; 95\% CI 1.0-6.0; Fig. 5). In addition, SARS-CoV-2 sero-prevalence decreased with increasing age group. It was lower in participants living in small apartment houses compared to participants living in single houses (OR adjusted for age and sex 0.0002; 95\% CI 0.0-0.14).

\section{Household and neighbourhood clustering of SARS-CoV-2 cases}

The analysis of potential household and neighbourhood clustering indicated a highly significant within-household clustering of SARS-CoV-2 cases. In contrast, no indications for neighbourhood transmission of SARS-CoV-2 were observed (Fig. 6, Additional file 1: Figure S5).

\section{Sensitivity analyses on behavioural factors and SARS-CoV-2 sero-incidence}

In order to identify which behavioural factors might be related to the different sero-prevalences in men and younger subjects, we compared these factors by sex and age group. The sum of contacts decreased significantly from the two younger age groups to the oldest age group in men and women ( $<<0.001$; Additional file 1: Table S4). In contrast, the self-estimated health-related risk taking behaviour and leisure time activity level were highest in

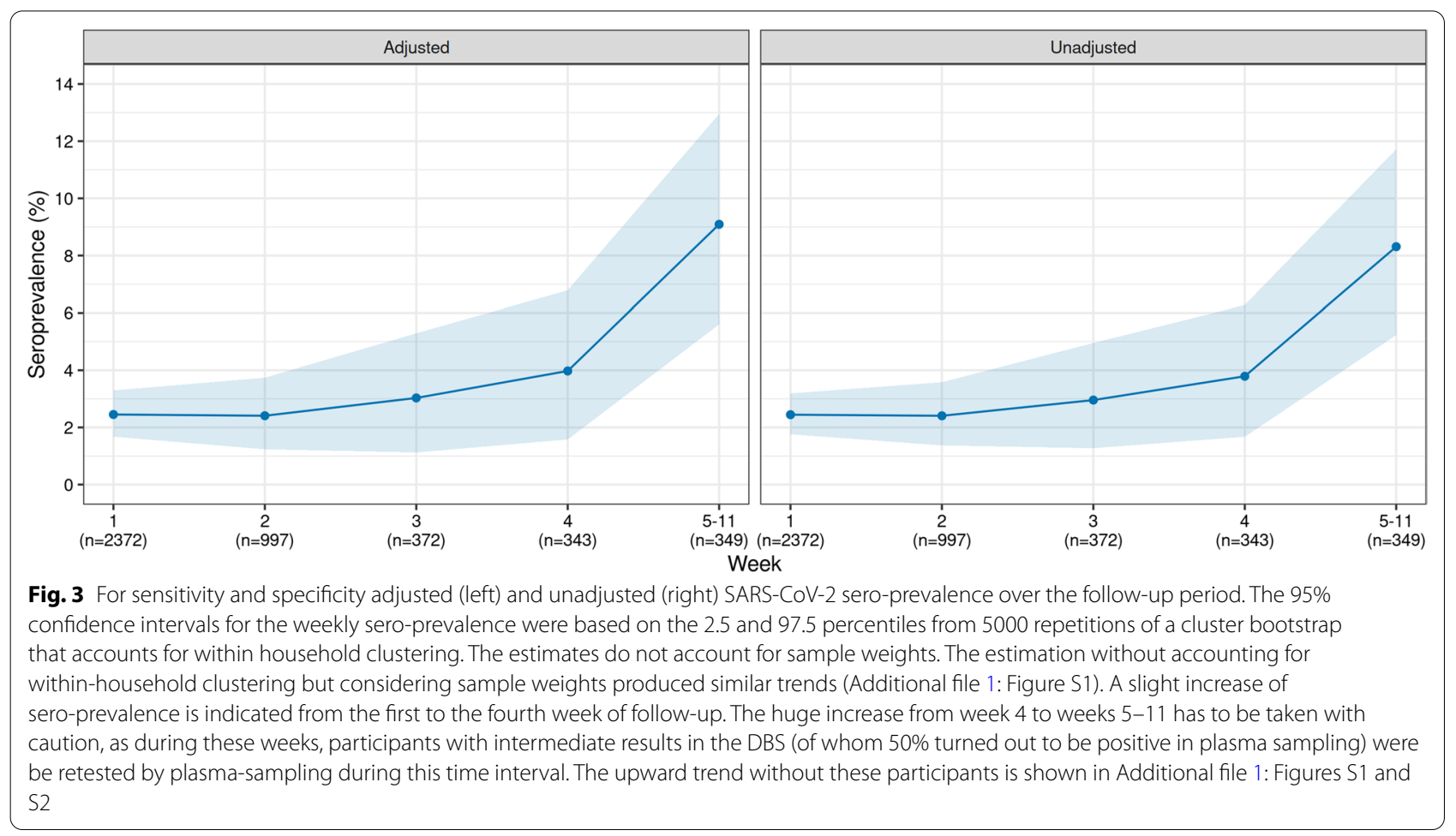



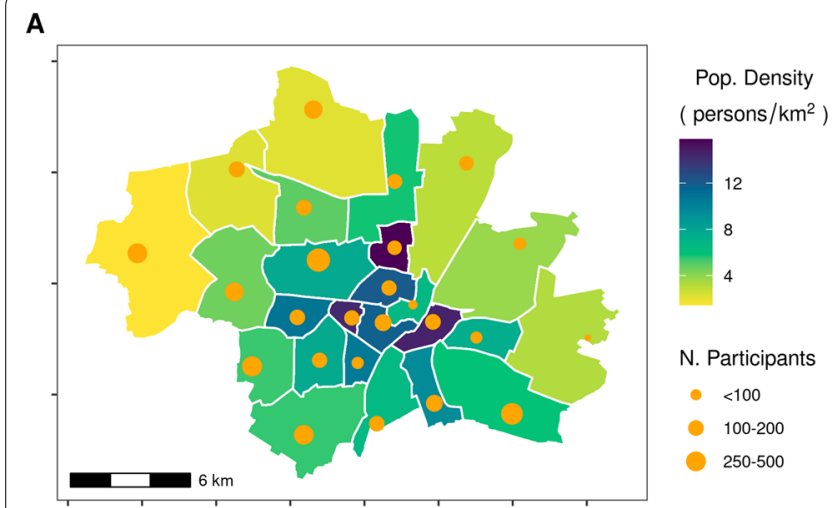

C

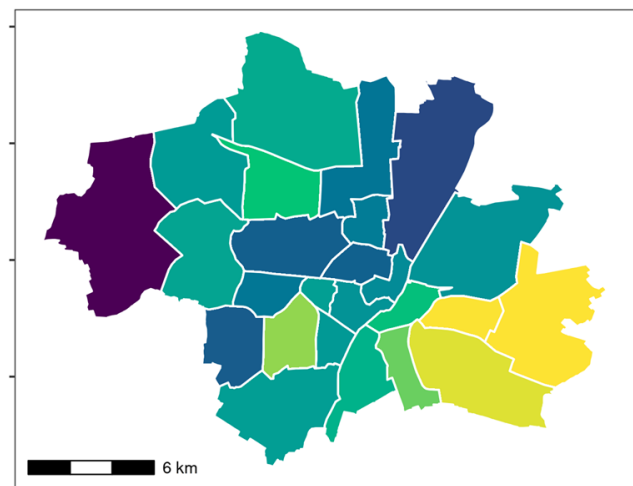

B

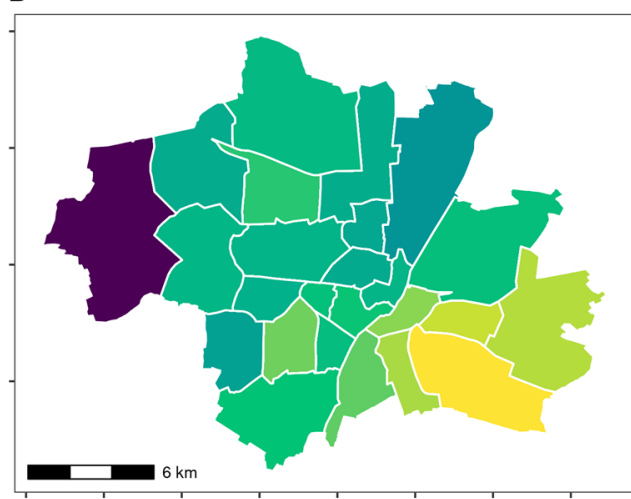

Prevalence (\%)

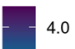

3.5

3.0

D

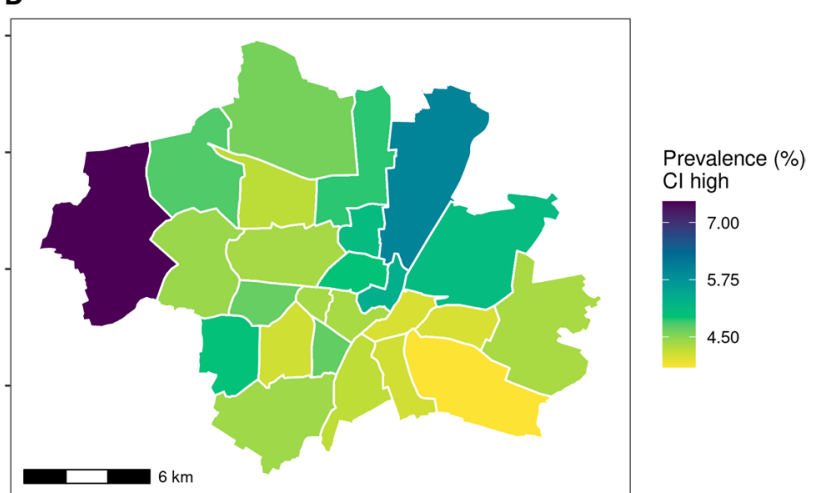

Fig. 4 Geospatial distribution of the crude SARS-CoV-2 sero-positivity across boroughs in Munich. A Population density (taken from https://simple. wikipedia.org/wiki/Boroughs_of_Munich) and number of participants in each city borough; B Weighted sample based SARS-CoV-2 sero-prevalence; C Lower 95\% confidence bounds of the weighted SARS-CoV-2 sero-prevalence; D Upper 95\% confidence bounds of the weighted SARS-CoV-2 sero-prevalence. The sero-positivity varied slightly across the boroughs (as indicated by different colours), however, differences did not reach statistical significance

the age group $<35$ years. Comparing men and women, self-estimated health-related risk taking behaviour was statistically higher for men than for women in the age group 35-65 years only $(\mathrm{p}<0.001$, Additional file 1 : Table S5). In contrast, sum of contacts and number of leisure time activities was similar for men and women by age strata $(\mathrm{p}>=0.05)$.

In order to check for effect modification by sex, age and behavioural factors, we calculated the sex- and agestratified SARS-CoV-2 sero-incidence between baseline and follow-up by self-estimated health-related risk taking behaviour (Fig. 7A), number of contacts outside own household (Fig. 7B), number of leisure time activities in summer 2020 (Fig. 7C). These data indicate a slightly higher risk of infection among men and women above the age of 34 years who indicated to have a high healthrelated risk taking behaviour compared to those with no high health-related risk taking behaviour. Men and women above the age of 64 years showed a higher SARS$\mathrm{CoV}-2$ sero-incidence if they had more personal contacts compared to participants having fewer personal contacts. Men with more leisure time activities in summer 2020 had a higher SARS-CoV-2 sero-incidence compared to less active men. However, all confidence intervals largely overlapped.

\section{Discussion}

Our data indicate a low SARS-CoV-2 sero-prevalence for the Munich general population living in private households eight months after the start of the pandemic. The incidence between the end of the first wave and the middle of the second wave was about as high as the related number of infections acquired during the first wave. Almost all sero-positive participants at baseline remained sero-positive at follow-up, indicating a high validity of the antibody test. Additionally, this supports previous reports suggesting that the humoral SARS-CoV-2 immune response is stable at least over the first eight months after infection [23, 24]. We also showed a predominance in SARS-CoV-2 sero-positivity among male 


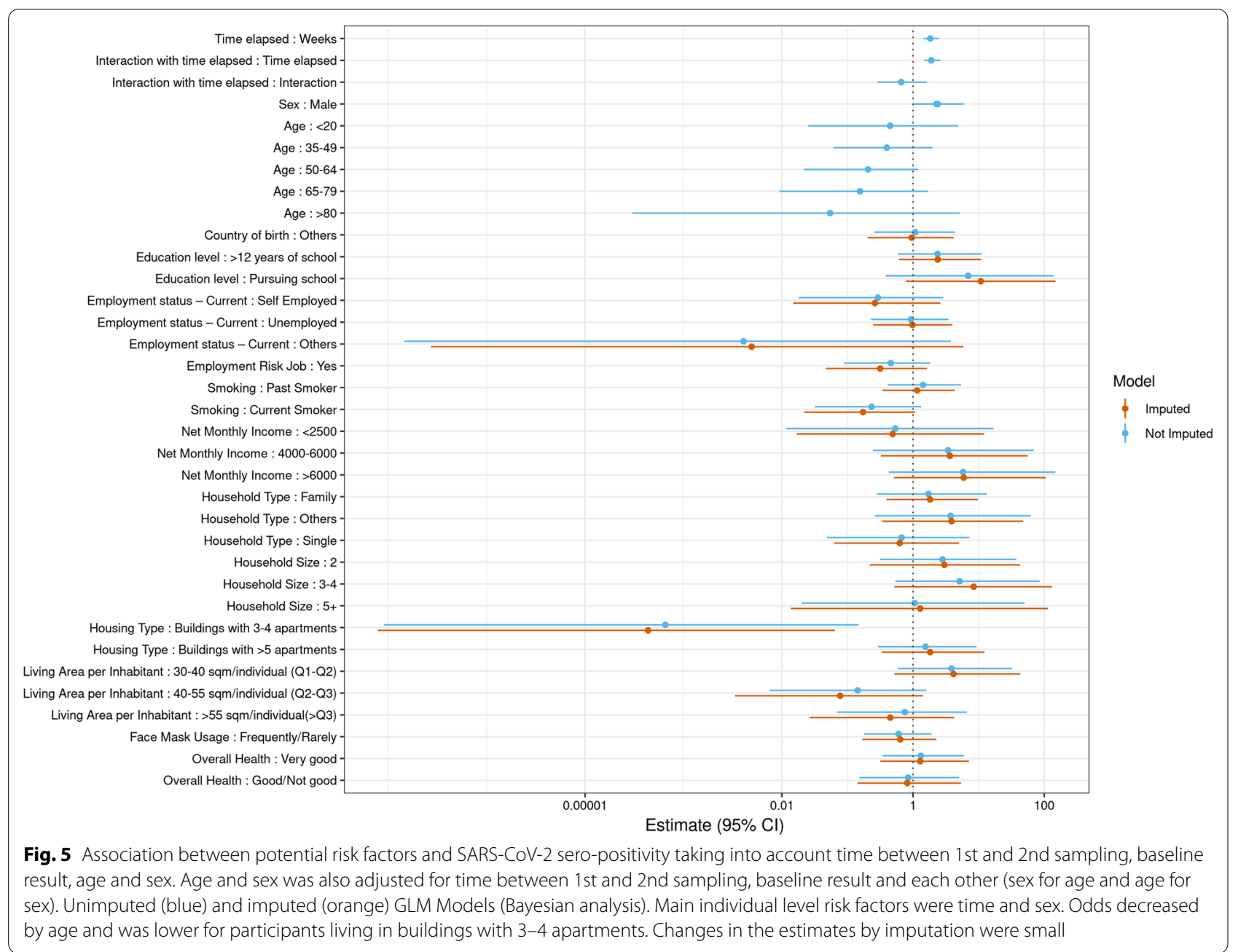

compared to female participants, and a reduced antibody prevalence with increasing age group.

Based on our data, the sero-prevalence for the Munich population above the age of 13 years living in private households was $3.6 \%$ (95\% CI $2.9-4.3 \%$ ). Until the end of November 2020, a total of 30,180 SARS-CoV-2 cases were officially registered in Munich (https://www.muenc hen.de/rathaus/Stadtinfos/Coronavirus-Fallzahlen.html\# Fallzahlen; Access date: 19-April-2021) which results in a population prevalence of $1.9 \%$. This prevalence increased to 44,377 registered cases by the end of December 2020 (population prevalence 3.0\%). The data are not directly comparable, as the official data also include children and persons living in institutions. While the prevalence of infection in children was at that time considered to be smaller than in adults, it was unknown for people living in institutions (e.g., homes for the elderly). Nevertheless, the comparison gives an indication that the percentage of officially registered infections improved considerably compared to the beginning of the pandemic.
In a previous publication we estimated that solely one out of four infections was registered by the official infectious diseases surveillance system [3]. A few population-based SARS-CoV-2 sero-studies have been conducted since the beginning of the pandemic (for review see [2]), most of them reporting sero-prevalences during or after the first wave. Up to now, only the Spanish national study reported the results of their follow-up data [25] with a sero-prevalence at follow-up (November 2020) of 5\%, and thus comparable to our results.

In our study, one predictor of change in sero-prevalence from baseline to follow-up was male sex. While a higher risk of more severe COVID-19 among men was confirmed in several studies [26], findings on sexdifferences in sero-prevalence are still inconsistent [2]. As younger age was also related to a larger increase in SARS-CoV-2 sero-prevalence at follow-up, one might assume that differences in behaviour may contribute to these findings. We could confirm differences in health-risk taking behaviour, frequency of leisure-time 


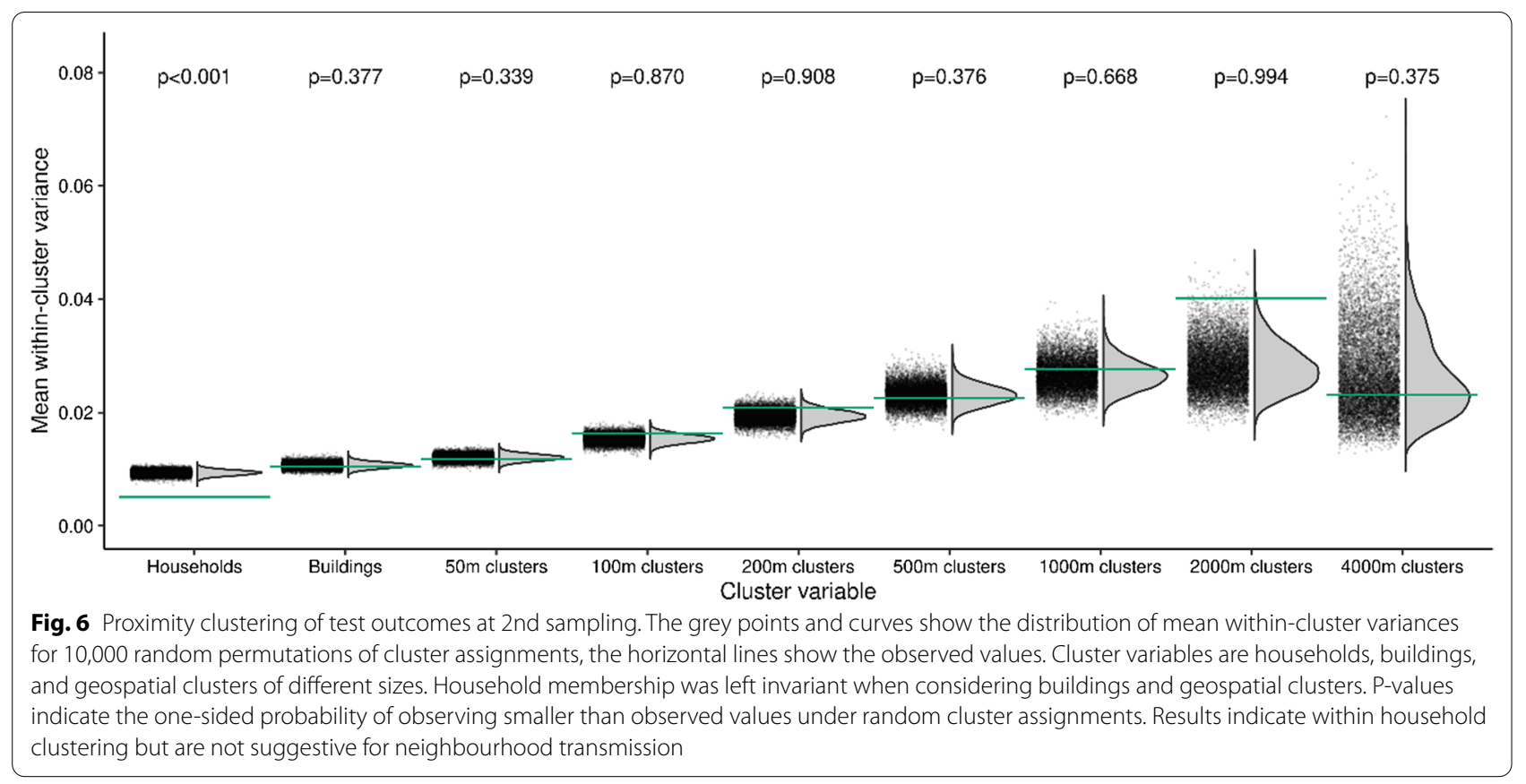

activities, and number of contacts outside the own household especially by age. In the stratified analyses of the incidence of infection by age and sex, we observed a tendency that behaviour is related to higher seroincidence of infection; although the low incidence and the reduced number of respondents to the questionnaires limited the statistical power of these analyses and result interpretation has to be done cautiously. However, the hypothesis that specific behaviour, i.e., restriction of contacts, might reduce the risk of infection is also supported by our observation that patients with autoimmune disease were at reduced risk of SARS-CoV-2 sero-positivity. This finding is in line with studies among, e.g., patients with inflammatory bowel disease [27]. Overall, our findings support the notion that behavioural factors contribute to the spread of the pandemic, and therefore actions to increase adherence to public-health measures (such as information campaigns) are crucial especially in a time when acceptance of measures in the general population is faltering.

Our results also confirm the importance of household clustering while no indications for neighbourhood clustering were seen. The former finding is also supported by the observation that participants from higher income households were at non-significantly higher odds of SARS-CoV-2 sero-positivity. As we took into account total household income (not adjusted for number of persons in the household), single households were more likely to be in the lower income category and thus, at lower likelihood of household transmission.
We also saw a non-significant trend for lower odds of SARS-CoV-2 antibodies in smokers compared to nonsmokers (OR 0.2; 95\% CI 0.02-1.1), confirming results of a meta-analysis [28]. Here, differences were mainly explained by differences in testing behaviour between smokers and non-smokers, which can be excluded in our study. One of the population-based studies published so far also indicated a lower SARS-CoV-2 sero-prevalence in smokers compared to non-smokers [29]. Whether this is a true effect of, e.g., nicotine [30] or vitamin D [31] or result of some form of bias needs to be evaluated in future studies. Of note is also the tendency for higher odds of SARS-CoV-2 in participants pursuing school, however, the wide confidence interval does not permit strong conclusions.

Among the strengths of our study are its populationbased, prospective nature in a large number of participants. Such population-based studies help authorities to plan public health measures based on the prevalence of exposure in the population, its spatial distribution and to further identify risk groups [32]. With increasing availability of vaccines, this study design with further followups will help public health authorities to understand the extent and duration of vaccine-induced immunity [33]. We previously showed a high sensitivity and specificity of the Elecsys ${ }^{\circledR}$ Anti-SARS-CoV-2 assay (Roche) used in this sero-study [5]. For the follow-up, we developed and carefully validated a semi-automated protocol using self-sampled DBS for SARS-CoV-2 serology [7]. This approach facilitates field work to a very considerable extent and 
A
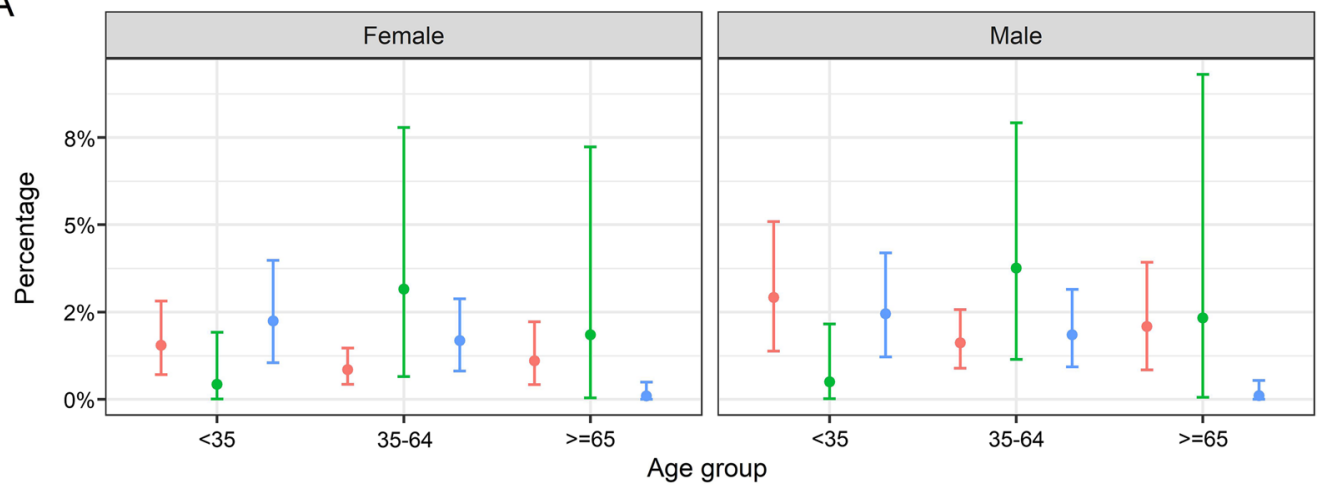

Risk perception for self-estimated health-related risk-taking behavior

$\rightarrow$ Not High $\rightarrow$ High $\rightarrow$ Missing

B

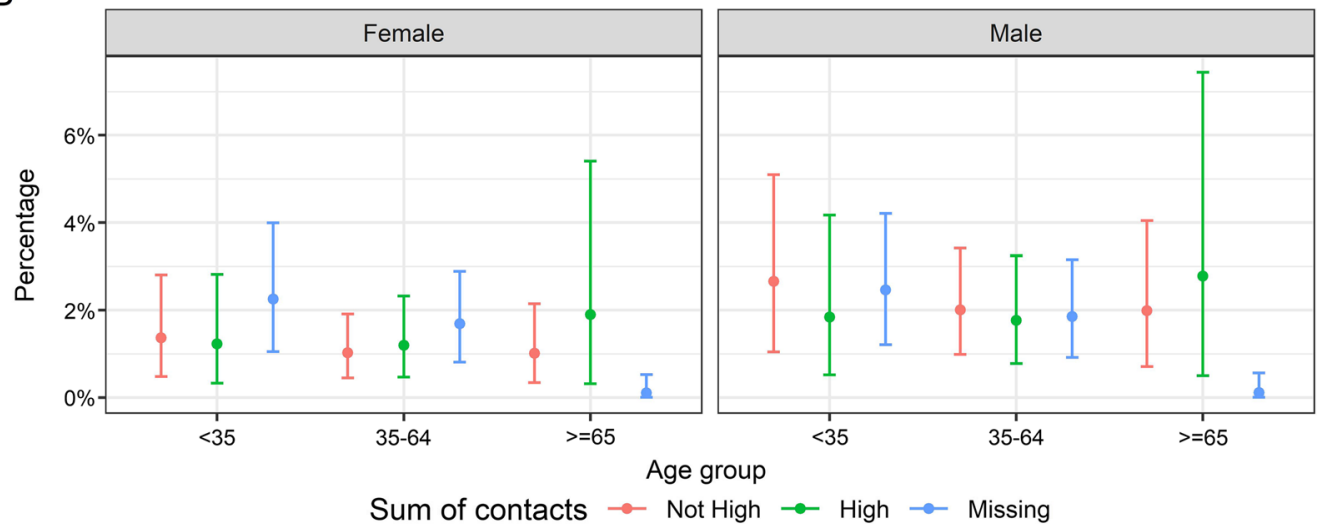

C

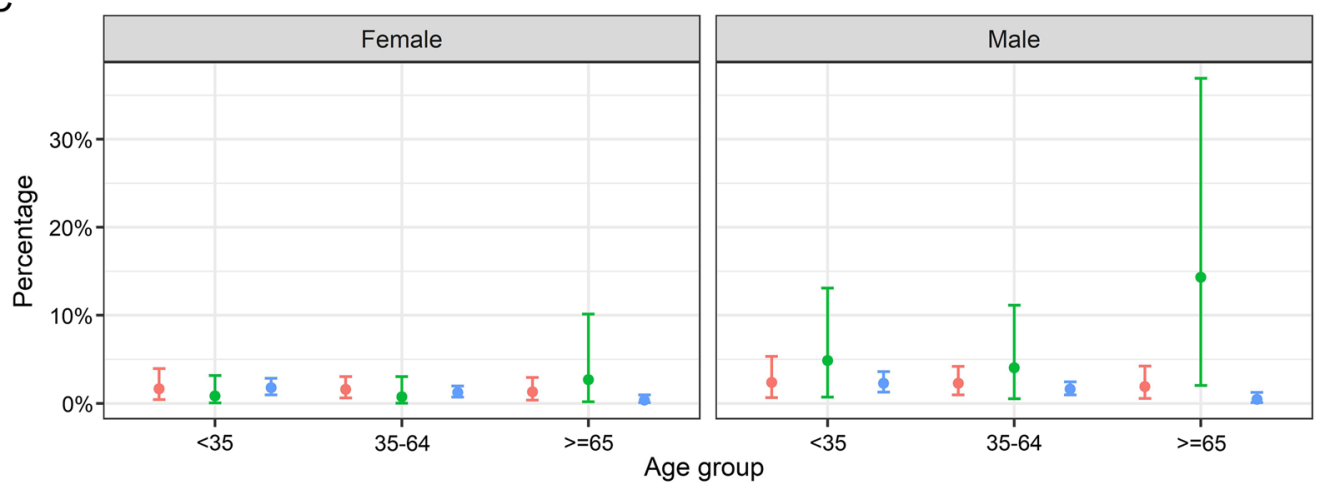

Activity level (Summer 2020) $\rightarrow$ Not High $\rightarrow$ High $\rightarrow$ Missing

Fig. 7 Sero-incidence of SARS-CoV-2 between baseline and follow-up by $\mathbf{A}$ self-estimated health-related risk-taking behaviour, B sum of contacts and $\mathbf{C}$ leisure time activities in summer 2020 stratified for sex and age group. Results suggest some role of behavioural factors in the risk of SARS-CoV-2 sero-positivity but differences are not statistically significant

thus, makes studies with a higher frequency of follow-ups more feasible. Acceptance was high in our study population, and the percentage of participants lost to follow-up comparably low.

However, in the analyses we had to take selective participation into account by modelling the underlying non-response mechanism and calibrating the weights. This way, we could reduce attrition bias in our prevalence and incidence estimates. It is common in prospective cohort studies that baseline participants in younger age groups, with migration background and with lower socioeconomic status are less likely to participate at follow-up 
[4]. While typically participants with positive outcome are also more likely to participate in follow-up studies (34), our baseline participants who were SARS-CoV-2 antibody positive were less likely to take part at followup. This gives some indication that unknown sero-status motivated at least part of our baseline participants to take part in the study. Once positive sero-status was known to them, they might have lost interest. Further supported is this hypothesis by the fact that less participants were willing to complete the follow-up questionnaires than to take part in the SARS-CoV-2 antibody follow-up. As a consequence, statistical power to analyse the association between behavioural factor and SARS-CoV-2 sero-positivity was limited. Finally, the age group 14 to 19 years is of specific interest given that data on sero-prevalence in this age group is still limited. However, the number of participants in this age group was low $(\mathrm{n}=212)$ and given the still low sero-prevalence at the time of the study, only 9 of them turned positive limiting the power of further analyses in this age group.

\section{Conclusions}

In conclusion, SARS-CoV-2 sero-prevalence in the Munich general population was still low by the end of 2020. Men and younger parts of the population were more likely to be affected. Risk-taking behaviour might be one reason for these differences. Therefore, non-pharmaceutical public health measures are still important.

\begin{abstract}
Abbreviations
95\% Cl: 95\% Confidence interval; KoCo19: Munich COVID-19 cohort; LMU: Ludwig-Maximilians-University; OR: Odds ratio; ORCHESTRA: Connecting European Cohorts to Increase Common and Effective Response to SARSCoV-2 Pandemic; SARS-CoV-2: Severe acute respiratory syndrome coronavirus type 2; Yrs: Years.
\end{abstract}

\section{Supplementary Information}

The online version contains supplementary material available at https://doi. org/10.1186/s12879-021-06589-4.

Additional file 1: Figure S1. For sensitivity and specificity adjusted (left) and unadjusted (right) SARS-CoV-2 sero-prevalence over the follow-up period excluding DBS intermediates. The $95 \%$ confidence intervals for the weekly sero-prevalence are based on the 2.5 and 97.5 percentiles from 5,000 repetitions of a cluster bootstrap that accounts for within household clustering. The estimates do not account for sample weights. Figure S2. Comparison of the sero-positivity over the follow-up period accounting for sampling weights. Figure S3. Sero-prevalence estimates across the different boroughs of Munich using CAR model priors with a single level of spatial autocorrelation as random effects. Figure S4. Sero-prevalence estimates across the different boroughs of Munich with CAR model priors through the dissimilarity of the population density in neighbouring boroughs for spatial autocorrelation. Figure S5. Distribution of mean within-cluster variance of test results under 10,000 random permutation of cluster assignments, with clusters being households, buildings, and geospatial clusters of different sizes. Household membership left invariant for building and geospatial clusters. Left: Value distribution. Right: 50\%,
95\%, 99\% Cls. Black lines (left) and dots (right) indicate the observed values. Table S1. Comparison of the follow-up plasma results, using DBS or Venous Blood Samples. Table S2. Comparison of the estimates for the covariate of population density at the constituency level using the Poisson and the negative binomial model. Table S3. Course of SARS-CoV-2 antibody status within the KoCo19 follow-up participants. Table S4. Summary across age group and sex for the response to the behaviour questionnaires stratified by sex. p-values are from Pearson's Chi-squared test with simulated $p$-value (based on 10000 replicates). Table S5. Summary across age group and sex for the response to the behaviour questionnaires stratified by age group. $\mathrm{p}$ - values are from Pearson's Chi-squared test with simulated $p$-value (based on 10000 replicates).

\section{Acknowledgements}

We gratefully thank all study participants for their trust, time, data, and specimens. This study would also not have been possible without the staff of the Division of Infectious Diseases and Tropical Medicine at the University Hospital of LMU Munich, Helmholtz Centre Munich, and Bundeswehr Institute of Microbiology, as well as all medical students involved. We thank Judith Eckstein for outstanding support regarding public relations. We thank the teams from the press offices of LMU, University Hospital of LMU Munich, and of Helmholtz Centre Munich. We thank the KoCo19 advisory board members Stefan Endres, Stephanie Jacobs, Bernhard Liebl, Michael Mihatsch, Matthias Tschöp, Manfred Wildner, and Andreas Zapf. We thank Accenture for the development of the KoCo19 web-based survey application. We are grateful to the Statistical Office of the City of Munich, Germany, for providing statistical data on the Munich general population and to Landesamt für Gesundheit und Lebensmittelsicherheit for carrying out laboratory measurements. We also thank Guillaume Chauvet for advice on the sampling design and variance estimation and Dr. Joachim Heinrich for advice on inclusion of population density. We are grateful to the Munich police for their support in the fieldwork of the baseline study. The Munich Surgical Imaging GmbH, Cisco Systems, and the graphic/photo/IT infrastructure departments at the University Hospital of LMU Munich provided support during video production and online events. For fieldwork of the baseline study, BMW Group as part of their campaign "BMW hilft Helfenden" provided free cars. Mercedes-Benz Munich provided support with Mercedes-Benz Rent in the project infrastructure. MG acknowledges the support from the Joachim Herz Foundation through the Add-on Fellowship for Interdisciplinary Science.

Members of the KoCo19 Study group: Nikolaus Ackermann, Emad Alamoudi, Jared Anderson, Maxilmilian Baumann, Marc Becker, Franziska Bednarzki, Olimbek Bemirayev, Patrick Bitzer, Rebecca Böhnlein, Friedrich Caroli, Josephine Coleman, Lorenzo Contento, Alina Czwienzek, Flora Deák, Jana Diekmannshemke, Gerhard Dobler, Jürgen Durner, Ute Eberle, Judith Eckstein, Tabea Eser, Philine Falk, Manuela Feyereisen, Volker Fingerle, Otto Geisenberger, Christof Geldmacher, Leonard Gilberg, Kristina Gillig, Philipp Girl, Elias Golschan, Elena Maria Guglielmini, Pablo Gutierrez, Anslem Haderer, Marlene Hannes, Lena Hartinger, Alejandra Hernandez, Leah Hillari, Christian Hinske, Tim Hofberger, Sacha Horn, Kristina Huber, Christian Janke, Ursula Kappl, Antonia Keßler, Zohaib Khan, Johanna Kresin, Arne Kroidl, Magdalena Lang, Clemens Lang, Silvan Lange, Michael Laxy, Reiner Leidl, Leopold Liedl, Xhovana Lucaj, Fabian Luppa, Alexandra Sophie Nafziger, Petra Mang, Alisa Markgraf, Rebecca Mayrhofer, Hannah Müller, Katharina Müller, Ivana Paunovic, Michael Plank, Claire Pleimelding, Stephan Prückner, Elba Raimúndez, Jakob Reich, Viktoria Ruci, Nicole Schäfer, Benedikt Schluse, Lara Schneider, Mirjam Schunk, Lars Schwettmann, Andreas Sing, Alba Soler, Peter Sothmann, Kathrin Strobl, Jeni Tang, Fabian Theis, Sophie Thiesbrummel, Vincent Vollmayr, Emilia von Lovenberg, Jonathan von Lovenberg, Julia Waibel, Claudia Wallrauch, Julia Wolff, Tobias Würfel, Houda Yaqine, Sabine Zange, Eleftheria Zeggini, Anna Zielke, Thorbjörn Zimmer.

\section{Authors' contributions}

M.H. is the principal investigator of this study and obtained most necessary funds. M.H., K.R. and E.S. conceived the study with input from M.N.D., F.F., J.M.G.N., I.K., L.O., M.PI., M.Pr., F.R., V.T. and A.W. Sample collection was led by J.M.G.N., I.K., D.M., L.O., M.Pr. and V.T. with assistance from I.B., J.B., J.F., G.F., K.P., C.R. and S.W. The laboratory set-up and sample processing were led by A.W., R.W. was responsible for virus micro-neutralization tests. M.N.D. coordinated the development of the web-based study application with input by E.S. 
M.N.D., F.F., K.R., F.R., E.S. and S.W. coordinated data acquisition and data management. Data was cleaned and prepared by N.C., M.N.D., F.F. and S.W. Statistical analyses and data visualization were performed by A.B., N.C., T.F., M.G., R.L.G., P.P. and Y.S. C.F. and J.H. led the statistical analyses. C.F., J.H., K.R., F.R. E.S. and S.W. conceptualized the result presentation with input from M.H., I.K., L.O., M.Pr. and A.W. The manuscript was primarily written by K.R. and A.B., with significant contributions from P.P., R.L.G., M.G., Y.S., A.W., M.H., J.H. and C.F. All authors have read and agreed to the published version of the manuscript.

\section{Funding}

Open Access funding enabled and organized by Projekt DEAL. This study was funded by the Bavarian State Ministry of Science and the Arts, the University Hospital of Ludwig-Maximilians-University Munich, the Helmholtz Centre Munich, the University of Bonn, the University of Bielefeld, the European Union's Horizon 2020 research and innovation programme (ORCHESTRA Grant agreement ID: 101016167), Munich Center of Health (McHealth), the Deutsche Forschungsgesellschaft (SEPAN Grant number: HA 7376/3-1), Volkswagenstiftung (E2 Grant number: 99450 ) and the German Ministry for Education and Research (MoKoCo19, reference number 01KI20271). Euroimmun, Mikrogen, Roche, and Viramed provided kits and machines for analyses at discounted rates. The funders had no role in study design, data collection, data analyses, data interpretation, writing, or submission of this manuscript.

\section{Availability of data and materials}

Our data are accessible to researchers upon reasonable request to the corresponding author taking data protection laws and privacy of study participants into account. To facilitate reproducibility and reuse, the analysis and figure generation code has been made available on GitHub (https://github.com/ koco19/epi2) and has been uploaded to ZENODO (https://doi.org/10.5281/ zenodo.4707037) for long-term storage.

\section{Declarations}

\section{Ethics approval and consent to participate}

The study was conducted in accordance with good clinical (GCP) and epidemiological practice (GEP) standards as well as the Declaration of Helsinki in its most recent form (as amended by the 64th WMA General Assembly, Fortaleza, Brazil, in October 2013). The study protocol was approved by the Institutional Review Board of the Medical Faculty at Ludwig Maximilian University Munich, Germany (opinion dated 31 March 2020; number 20-275; opinion date amendment: 10 October 2020), prior to study initiation. Written informed consent was obtained from all study participants prior to study inclusion.

\section{Consent for publication}

Not applicable as no identifiable information was included in the manuscript.

\section{Competing interests}

In addition to the funding disclosed in the funding section, DM and VT are sub-investigators vaccines trials sponsored by Curevac AG. LO received non-financial support from Dr. Box Betrobox and grants from the Bavarian State Ministry of Science and the Arts during the conduct of the study. CR received royalities from Elsevier LTD for the textbook "Clinical Cases in Tropical Medicine". She also received honoraria for lectures done at non-private academic institutions. CR is also chair of the "Travel medicine" board of the of the German Society for the Tropical Medicine, Travel Medicine and global health (unpaid) and member of the permanent committee on vaccinations at the Robert-Koch-Institute work group on travel vaccinations (unpaid). AW is on Roche Diagnostics and Roche Pharma Advisory Boards related SARS-CoV-2. The Bundeswehr Institute of Microbiology (RW) received research funding from the Medical Biodefence program of the Bundeswehr. The funders had no role in study design, data collection, data analyses, data interpretation, writing, or submission of this manuscript.

\section{Author details}

${ }^{1}$ Institute and Outpatient Clinic for Occupational, Social and Environmental Medicine, University Hospital, LMU Munich, 80336 Munich, Germany. ${ }^{2}$ Center for International Health (CIH), University Hospital, LMU Munich, 80336 Munich, Germany. ${ }^{3}$ Comprehensive Pneumology Center (CPC) Munich, German Center for Lung Research (DZL), 89337 Munich, Germany. ${ }^{4}$ Division of Infectious Diseases and Tropical Medicine, University Hospital, LMU Munich, 80802 Munich,
Germany. ${ }^{5}$ Institute of Computational Biology, Helmholtz Zentrum MünchenGerman Research Center for Environmental Health, 85764 Neuherberg, Germany. ${ }^{6}$ Faculty of Business Administration and Economics, Bielefeld University, 33615 Bielefeld, Germany. ${ }^{7}$ Core Facility Statistical Consulting, Helmholtz Zentrum München-German Research Center for Environmental Health, 85764 Neuherberg, Germany. ${ }^{8}$ German Center for Infection Research (DZIF), partner site, Munich, Germany. ${ }^{9}$ Center for Mathematics, Technische Universität München, 85748 Garching, Germany. ${ }^{10}$ Bundeswehr Institute of Microbiology, 80937 Munich, Germany. ${ }^{11}$ Interdisciplinary Research Unit Mathematics and Life Sciences, University of Bonn, 53113 Bonn, Germany.

Received: 16 May 2021 Accepted: 19 August 2021

Published online: 08 September 2021

\section{References}

1. Rostami A, Sepidarkish M, Leeflang MMG, Riahi SM, Nourollahpour Shiadeh M, Esfandyari S, et al. SARS-CoV-2 seroprevalence worldwide: a systematic review and meta-analysis. Clin Microbiol Infect. 2021;27(3):331-40.

2. Lai CC, Wang JH, Hsueh PR. Population-based seroprevalence surveys of anti-SARS-CoV-2 antibody: an up-to-date review. Int J Infect Dis. 2020;101:314-22.

3. Pritsch M, Radon K, Bakuli A, Le Gleut R, Olbrich L, Guggenbuhl Noller JM, et al. Prevalence and risk factors of infection in the representative COVID19 cohort Munich. Int J Environ Res Public Health. 2021. https://doi.org/ 10.3390/ijerph18073572.

4. Radon K, Saathoff E, Pritsch M, Guggenbuhl Noller JM, Kroidl I, Olbrich L, et al. Protocol of a population-based prospective COVID-19 cohort study Munich, Germany (KoCo19). BMC Public Health. 2020;20(1):1036.

5. Olbrich L, Castelletti N, Schälte Y, Garí M, Pütz P, Bakuli A, et al. A serology strategy for epidemiological studies based on the comparison of the performance of seven different test systems - the representative COVID19 cohort Munich. medRxiv. 2021;26:1033.

6. Iglesias C, Torgerson D. Does length of questionnaire matter? A randomised trial of response rates to a mailed questionnaire. J Health Serv Res Policy. 2000;5(4):219-21.

7. Beyerl J, Rubio-Acero R, Castelletti N, Panovic I, Kroidl I, Khan ZN, et al. A dried blood spot protocol for high throughput analysis of SARSCoV-2 Serology based on the Roche Elecsys anti-N assay. EBioMedicine. 2021;70:103502.

8. Sempos CT, Tian L. Adjusting coronavirus prevalence estimates for laboratory test kit error. Am J Epidemiol. 2021;190(1):109-15.

9. Särndal CE, Swensson B, Wretman J. Model assisted survey sampling. New York: Springer; 2003.

10. Deville JC, Särndal CE. Calibration estimators in survey sampling. J Am Stat Assoc. 1992:87(418):376-82.

11. Deville JC. Variance estimation for complex statistics and estimators: linearization and residual techniques. Surv Meth. 1999;25(2):193-204.

12. Juillard $\mathrm{H}$, Chauvet $\mathrm{G}$. Variance estimation under monotone nonresponse for a panel survey. Surv Meth. 2018;44(2). Paper available at https//www150.statcan.gc.ca/n1/pub/12-001-x/2018002/article/54952eng.htm.

13. Cameron AC, Gelbach JB, Miller DL. Bootstrap-based improvements for inference with clustered errors. Rev Econ Stat. 2008;90(3):414-27.

14. Nichols TE, Holmes AP. Nonparametric permutation tests for functional neuroimaging: a primer with examples. Hum Brain Mapp. 2002;15(1):1-25.

15. Lee D. CARBayes: an R package for Bayesian spatial modeling with conditional autoregressive priors. J Stat Softw. 2013;55(13):1-24.

16. Leroux $B$, Lei $X$, Breslow N. Estimation of disease rates in small areas: a new mixed model for spatial dependence. In: Halloran ME, Berry D, editors. Models in epidemiology, the environment and clinical trials. New York: Springer; 1999. p. 135-78.

17. Lee D, Mitchell R. Boundary detection in disease mapping studies. Biostatistics. 2012;13(3):415-26.

18. Lee D. A comparison of conditional autoregressive models used in Bayesian disease mapping. Spat Spatiotemporal Epidemiol. 2011;2(2):79-89.

19. Bürkner P. Advances Bayesian multilevel modeling with the $R$ package brms. R J. 2018;10(1):395-411. 
20. Bürkner P. brms: an R package for Bayesian multilevel models using stan. J Stat Softw. 2017;80(1):1-28.

21. Erler NS, Rizopoulos D, Lesaffre EM. JointAl: joint analysis and imputation of incomplete data in R. J Stat Softw. 2020; arXiv:1907.10867.

22. Erler NS, Rizopoulos D, Rosmalen J, Jaddoe VW, Franco OH, Lesaffre EM. Dealing with missing covariates in epidemiologic studies: a comparison between multiple imputation and a full Bayesian approach. Stat Med. 2016;35(17):2955-74.

23. Dan JM, Mateus J, Kato Y, Hastie KM, Yu ED, Faliti CE, et al. Immunological memory to SARS-CoV-2 assessed for up to 8 months after infection. Science. 2021. https://doi.org/10.1126/science.abf4063.

24. He Z, Ren L, Yang J, Guo L, Feng L, Ma C, et al. Seroprevalence and humoral immune durability of anti-SARS-CoV-2 antibodies in Wuhan, China: a longitudinal, population-level, cross-sectional study. Lancet. 2021;397(10279):1075-84.

25. Pérez-Olmeda M, Saugar JM, Fernández-García A, Pérez-Gómez B, Pollán $M$, Avellón A, et al. Evolution of antibodies against SARS-CoV-2 over seven months: experience of the Nationwide Seroprevalence ENE-COVID Study in Spain. Nat Commun. 2021;12(1):4740.

26. Takahashi T, Ellingson MK, Wong P, Israelow B, Lucas C, Klein J, et al. Sex differences in immune responses that underlie COVID-19 disease outcomes. Nature. 2020;588(7837):315-20.

27. Stallmach A, Sturm A, Blumenstein I, Helwig U, Koletzko S, Lynen P, et al. Addendum to S3-Guidelines Crohn's disease and ulcerative colitis: management of patients with inflammatory bowel disease in the COVID-19 pandemic —open questions and answers. Z Gastroenterol. 2020:58(10):982-1002.
28. Simons D, Shahab L, Brown J, Perski O. The association of smoking status with SARS-CoV-2 infection, hospitalization and mortality from COVID-19: a living rapid evidence review with Bayesian meta-analyses (version 7). Addiction. 2021;116(6):1319-68.

29. Carrat F, de Lamballerie X, Rahib D, Blanché H, Lapidus N, Artaud F, et al. Antibody status and cumulative incidence of SARS-CoV-2 infection among adults in three regions of France following the first lockdown and associated risk factors: a multicohort study. Int J Epidemiol. 2021;dyab1 10

30. Farsalinos K, Niaura R, Le Houezec J, Barbouni A, Tsatsakis A, Kouretas D, et al. Editorial: Nicotine and SARS-CoV-2: COVID-19 may be a disease of the nicotinic cholinergic system. Toxicol Rep. 2020;7:658-63.

31. Khan AH, Nasir N, Nasir N, Maha Q, Rehman R. Vitamin D and COVID-19: is there a role? J Diabetes Metab Disord. 2021;20(1):1-8. https://doi.org/10. 1007/s40200-021-00775-6.

32. Peeling RW, Olliaro PL. The time to do serosurveys for COVID-19 is now. Lancet Respir Med. 2020;8(9):836-8.

33. Eckerle I, Meyer B. SARS-CoV-2 seroprevalence in COVID-19 hotspots. Lancet. 2020;396(10250):514-5.

34. Forster F, KreissI S, Wengenroth L, Vogelberg C, von Mutius E, Schaub B, et al. Third follow-up of the study on occupational allergy risks (SOLAR III) in Germany: design, methods, and initial data analysis. Front Public Health. 2021;9: 591717

\section{Publisher's Note}

Springer Nature remains neutral with regard to jurisdictional claims in published maps and institutional affiliations.
Ready to submit your research? Choose BMC and benefit from:

- fast, convenient online submission

- thorough peer review by experienced researchers in your field

- rapid publication on acceptance

- support for research data, including large and complex data types

- gold Open Access which fosters wider collaboration and increased citations

- maximum visibility for your research: over 100M website views per year

At BMC, research is always in progress.

Learn more biomedcentral.com/submissions 